\title{
Low circulating dihomo-gamma-linolenic acid is associated with diabetic retinopathy: a cross sectional study of KAMOGAWA-DM cohort study
}

\author{
Takuro Okamura ${ }^{1)}$, Hanako Nakajima ${ }^{1)}$, Yoshitaka Hashimoto ${ }^{1)}$, Saori Majima ${ }^{1)}$, Takafumi Senmaru ${ }^{1)}$, \\ Emi Ushigome ${ }^{1)}$, Naoko Nakanishi ${ }^{1)}$, Masahide Hamaguchi ${ }^{1)}$, Mai Asano ${ }^{1)}$, Masahiro Yamazaki ${ }^{1)}$, \\ Hiroshi Takakuwa ${ }^{2)}$ and Michiaki Fukui ${ }^{1)}$

\footnotetext{
1) Department of Endocrinology and Metabolism, Kyoto Prefectural University of Medicine, Kyoto 602-8566, Japan

2) Agilent Technologies, Chromatography Mass Spectrometry Sales Department, Life Science and Applied Markets Group, Tokyo 192-8510, Japan
}

\begin{abstract}
Diabetic retinopathy (DR), one of the major complications of diabetes, can cause blindness and reduce quality of life. Dyslipidemia is reported to be associated with DR, whereas arachidonic acid may have a protective effect against DR. We aimed to investigate the association of circulating n-3 and n-6 polyunsaturated fatty acids (PUFAs) with DR. In this crosssectional study, 190 Japanese patients with type 2 diabetes were classified as no diabetic retinopathy (NDR), simple diabetic retinopathy (SDR), or proliferative diabetic retinopathy (PDR) including pre-proliferative diabetic retinopathy. Circulating fatty acids (FAs) were measured by gas chromatograph-mass spectrometry. Logistic regression analysis was performed to investigate the association between the levels of FAs and the presence of DR. The average age, body mass index and the duration of diabetes were $62.7 \pm 12.1$ years, $25.0 \pm 4.5 \mathrm{~kg} / \mathrm{m}^{2}$, and $9.8 \pm 8.7$ years, respectively. Twenty-seven patients were diagnosed with DR. Circulating levels of dihomo-gamma-linolenic acid (DGLA) in the NDR $(n=163)$, SDR $(n=13)$ and PDR $(n=14)$ groups were $28.3 \pm 11.0 \mu \mathrm{g} / \mathrm{mL}, 24.4 \pm 9.7 \mu \mathrm{g} / \mathrm{mL}$, and $21.8 \pm 6.2 \mu \mathrm{g} / \mathrm{mL}$, respectively $(p=0.032)$. The logarithm of circulating DGLA levels was associated with the presence of DR after adjusting for covariates (OR of 1-unit increment: $0.79,95 \%$ CI: $0.62-1.00, p=0.049)$. Circulating DGLA was negatively associated with the presence of DR.
\end{abstract}

Key words: Cohort study, Epidemiology, Fatty acids, Dihomo-gamma-linolenic acid, Diabetic retinopathy

DIABETIC RETINOPATHY (DR), a common microvascular complication of diabetes that causes irreversible retinal damage, is the leading cause of vision loss among working-age adults. Approximately one-third of patients with diabetes have some degrees of DR, and one in ten will develop a vision-threatening disease $[1,2]$. Therefore, the prevention and treatment of DR is one of the most important issues in the treatment of diabetes.

Dyslipidemia is correlated with the progression of DR in humans [3]. In the Early Treatment Diabetic Retinopathy Study, dyslipidemia increased the risk of developing hard exudates in the macula and visual loss [4]. Excess free fatty acid metabolism results in dyslipidemia, and fatty

Submitted Sep. 7, 2020; Accepted Nov. 4, 2020 as EJ20-0564 Released online in J-STAGE as advance publication Dec. 22, 2020 Correspondence to: Yoshitaka Hashimoto, Department of Endocrinology and Metabolism, Kyoto Prefectural University of Medicine, Graduate School of Medical Science, 465, Kajii-cho, Kamigyo-ku, Kyoto City, Kyoto 621-8585, Japan.

E-mail: y-hashi@koto.kpu-m.ac.jp acids (FAs) are also involved in inflammation and related chronic diseases $[5,6]$. Circulating FAs are composed of saturated FAs with no double bonds, monounsaturated FAs with a single double bond, and polyunsaturated FAs (PUFAs) with multiple double bonds. The association between FAs and DR has been reported in several studies, with early research suggesting a protective role of PUFAs against DR [7, 8], and recent reports associating n-3 PUFAs with a reduced risk of DR [9, 10]. In several animal studies, an n-3 PUFA-enriched diet was protective of retinal neuron function $[11,12]$. While a protective role of n-3 PUFAs against DR has been observed, there has been no significant association reported to date between n-6 PUFAs and DR. Gamma-linolenic acid (GLA) and dihomo-gamma-linolenic acid (DGLA) have been shown to induce T-regulatory cell activity and reduce the production of proinflammatory interleukin-1 and tumor necrosis factor-alpha [13]. In addition, moderate intakes of dietary n-6 PUFAs have reduced the risk of coronary heart disease [14], while circulating n-6 PUFAs 
are reported to be inversely associated with total and coronary heart disease mortality [15]. Taken together, not only n-3 PUFAs but also n-6 PUFAs have demonstrated anti-inflammatory effects.

In recent years, it is clearly shown that of patients with diabetes have different circulating FAs compared to those in people without diabetes, which may contribute to the development of organ damages [16-18]. Nevertheless, the association between these circulating FAs, especially detailed composition of FAs, and diabetic retinopathy has remained unclear. Therefore, this crosssectional study investigated the association between circulating n-3 or n-6 PUFAs and DR.

\section{Materials and Methods}

\section{Study design and participants}

The KAMOGAWA-DM study is an ongoing prospective cohort study that began in 2014 and includes the outpatient clinics of the Kyoto Prefectural University of Medicine (Kyoto, Japan) and the Kameoka Municipal Hospital (Kameoka, Japan) [19]. For the present crosssectional study, we included patients with T2DM whose circulating FAs were measured, and excluded those who were taking eicosatetraenoic acid supplements. T2DM was diagnosed at all participating institutions based on the criteria by the American Diabetes Association [20]. This study was approved by the Ethics Committee of each participating hospital (ERB-C-534, RBMRE-466-5).

\section{Data collection}

Information about patients' background demographics (i.e., age, sex, disease duration, and smoking habits) was gathered from their medical records. Blood pressure was measured in the outpatient clinics. After an overnight fast, venous blood samples were collected to measure fasting plasma levels of glucose, C-peptide, triglycerides, total cholesterol, high-density lipoprotein cholesterol, low-density lipoprotein cholesterol, creatinine, and uric acids. The level of hemoglobin A1c (HbA1c) was determined by high-performance liquid chromatography and presented herein using the National Glycohemoglobin Standardization Program unit.

Diagnosis of DR was made by ophthalmologists based on the Davis classification: no diabetic retinopathy (NDR), simple diabetic retinopathy (SDR), or proliferative diabetic retinopathy (PDR) that also includes preproliferative diabetic retinopathy. Patients with DR were defined as those with SDR or PDR.

\section{Estimation and assessment of the patients' habitual food and nutrient intake}

A brief-type self-administered diet history questionnaire (BDHQ) was used for the assessment of habitual food and nutrient intakes [21]. Details of the BDHQ were provided elsewhere [22]. Briefly, the BDHQ is a dietary recall to assess the respondent's dietary intakes of 58 items over the past month. Based on the Standard Tables of Food Composition in Japan, we estimated the intakes of total energy (kcal/day), carbohydrate (g/day), protein (g/day), and total fat (g/day) using the calculation algorithm of BDHQ [22].

\section{Measurement of circulating free fatty acids}

The composition of FAs in frozen serum samples was measured by gas chromatograph-mass spectrometry (GC/MS) with the Agilent 7890B/5977B system (Agilent Technologies, Santa Clara, CA, USA). Fatty acids compositions can be measured by several ways. In this study, we chose the serum, because it was the simplest method and could be applied clinically. Twenty-five $\mu \mathrm{L}$ of serum was methylated with a fatty acid methylation kit (Nacalai Tesque, Kyoto, Japan). The final product was loaded onto a Varian capillary column (DBFATWAX UI; Agilent Technologies). The capillary column used for fatty acid separation was CP-Sil 88 for FAME (100 $\mathrm{m}$ with an inner diameter of $0.25 \mathrm{~mm}$ and a membrane thickness of $0.20 \mu \mathrm{m}$, Agilent Technologies). The column temperature was maintained at $100^{\circ} \mathrm{C}$ for 4 min, increased gradually by $3^{\circ} \mathrm{C} / \mathrm{min}$ to $240^{\circ} \mathrm{C}$, and maintained at $240^{\circ} \mathrm{C}$ for $7 \mathrm{~min}$. The sample was injected in the split mode with a split ratio of 5:1. Each fatty acid methyl ester was detected in selected ion monitoring mode. All results were normalized to the peak height of the internal standard C17:0 [23]. Average CV was $0.0245 \%$ (range $0.004-0.039 \%)(n=3)$ for intra-day precision during a single analytical run and average CV $0.00237 \%(0.0010-0.0033 \%)$ on two separated days $(n=6)$ for inter-day precision. Each analytical run included a blank sample (without internal standard), and standard concentrations for calibration, and replicate sets of QC samples: $0.56 \mu \mathrm{g} / \mathrm{mL}, 1.77 \mu \mathrm{g} / \mathrm{mL}, 11.22 \mu \mathrm{g} / \mathrm{mL}$, and $60.00 \mu \mathrm{g} / \mathrm{mL}$ and found to be linear from the concentrations of 0.56 to $60.00 \mu \mathrm{g} / \mathrm{mL}$. $(\mathrm{y}=0.159 \mathrm{x}+0.1392)$.

\section{Statistical analysis}

The JMP program (ver. 13.0, SAS Institute, Cary, NC) was used for all statistical analyses. Differences with $p$ values $<0.05$ were considered significant. Categorical variables were expressed as frequencies, and continuous variables were presented as mean \pm standard deviation (SD). The Chi-square test for categorical variables and the Wilcoxon signed-rank test for continuous variables 
were used to assess the differences between groups. The difference in each circulating FA level among patients with NDR, SDR, and PDR was compared by one-way ANOVA and Tukey's honestly significant difference test. Circulating DGLA levels were log transformed to create a more normal distribution. The odds ratio (OR) and $95 \%$ confidence interval (CI) of the logarithm of circulating DGLA levels and other variables based on the presence of DR were assessed by univariate and multivariate logistic regression analyses adjusting for sex, disease duration [24], systolic blood pressure, HbAlc [25], triglycerides, total cholesterol, high-density lipoprotein cholesterol [26], omega-6 fatty acids [27, 28], smoking status [29], total energy intake [30], and statin usage [31].

\section{Results}

Among 426 patients (234 males and 192 females) with T2DM who completed the BDHQ, circulating FAs were measured in 197 patients (96 males and 101 females). Seven patients were excluded due to the use of eicosapentaenoic acid supplements (Fig. 1).

The clinical characteristics of the 190 patients included in this study are summarized in Table 1 . The average age and the level of $\mathrm{HbAlc}$ was $62.7 \pm 12.1$ years and $7.5 \pm 1.6 \%$, respectively. The frequency (percentage) of patients with NDR, SDR, and PDR was 163 (85.8\%), $13(6.8 \%)$, and $14(7.4 \%)$, respectively.

The differences of circulating PUFAs among diabetic retinopathy were evaluated (Table 2, Fig. 2). There was a difference of DGLA levels among retinopathy.

DGLA was negatively associated with the presence of $\mathrm{DR}$ in both the univariate logistic regression analysis (OR of 1-unit increment: $0.76,95 \%$ CI: $0.62-0.96, p=$ 0.020 ) and multivariate logistic regression analysis after

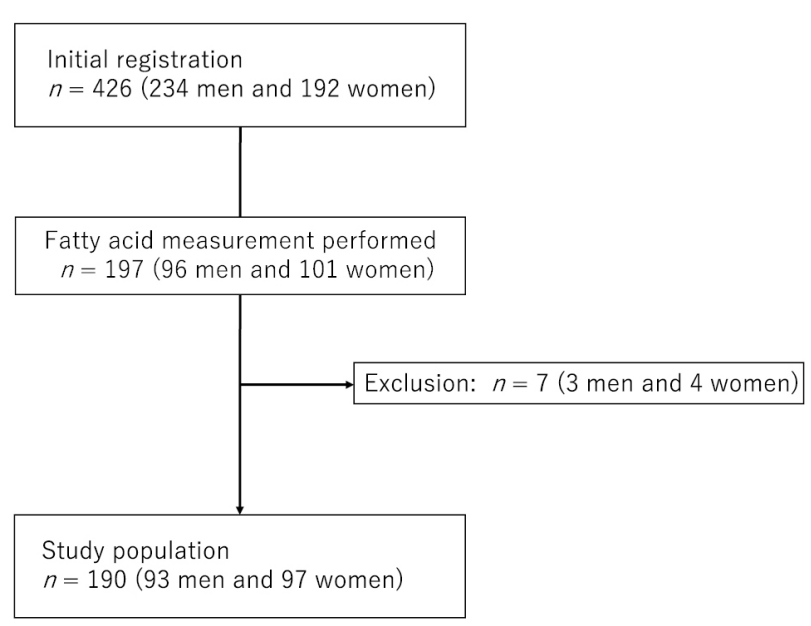

Fig. 1 The registration of patients. adjusting for covariates (OR of 1-unit increment: 0.73 , 95\% CI: $0.53-0.99, p=0.043$ ) (Table 3).

Table 1 Clinical characteristics of the study patients

\begin{tabular}{|c|c|}
\hline & Total $(n=190)$ \\
\hline Sex, men/women & $93 / 97$ \\
\hline Age, yrs & $62.7(12.1)$ \\
\hline Duration of diabetes, yrs & $9.8(8.7)$ \\
\hline BMI, $\mathrm{kg} / \mathrm{m}^{2}$ & $25.0(4.5)$ \\
\hline Systolic blood pressure, $\mathrm{mmHg}$ & $135.6(19.1)$ \\
\hline Diastolic blood pressure, $\mathrm{mmHg}$ & $81.8(10.7)$ \\
\hline Fasting plasma glucose, $\mathrm{mmol} / \mathrm{L}$ & $7.2(1.6)$ \\
\hline Hemoglobin A1c, \% & $7.5(1.6)$ \\
\hline C-peptide, $\mathrm{nmol} / \mathrm{L}$ & $0.6(0.3)$ \\
\hline Triglycerides, mmol/L & $1.5(0.9)$ \\
\hline Total cholesterol, $\mathrm{mmol} / \mathrm{L}$ & $5.2(1.0)$ \\
\hline High-density lipoprotein cholesterol, $\mathrm{mmol} / \mathrm{L}$ & $1.5(0.5)$ \\
\hline Low-density lipoprotein cholesterol, $\mathrm{mmol} / \mathrm{L}$ & $3.0(0.7)$ \\
\hline Creatinine, $\mu \mathrm{mol} / \mathrm{L}$ & $70.9(22.0)$ \\
\hline Estimated GFR, $\mathrm{mL} / \mathrm{min} / 1.73 \mathrm{~m}^{2}$ & $70.4(18.5)$ \\
\hline Uric acids, $\mathrm{mmol} / \mathrm{L}$ & $309.8(77.7)$ \\
\hline Urine albumin to creatinine ratio, $\mathrm{mg} / \mathrm{gCr}$ & $202.0(583.0)$ \\
\hline \multicolumn{2}{|l|}{ Diabetic nephropathy } \\
\hline Normoalbuminuria & $115(60.5)$ \\
\hline Microalbuminuria & $49(25.8)$ \\
\hline Macroalbuminuria & $26(13.7)$ \\
\hline \multicolumn{2}{|l|}{ Diabetic retinopathy } \\
\hline NDR & $163(85.8)$ \\
\hline SDR & $13(6.8)$ \\
\hline PDR & $14(7.4)$ \\
\hline Diabetic neuropathy $-/+$ & $150 / 40$ \\
\hline Never smoker & $120(63.2)$ \\
\hline Ex-smoker & $39(20.5)$ \\
\hline Current smoker & $31(16.3)$ \\
\hline Energy intake/day, kcal & $1,682.5(587.3)$ \\
\hline Carbohydrate intake, $\mathrm{g} /$ day & $209.9(80.6)$ \\
\hline Protein intake, $g /$ day & $71.7(28.6)$ \\
\hline Total fat intake, $g$ /day & $53.5(20.0)$ \\
\hline
\end{tabular}

Data are expressed as number (\%) of subjects or mean (SD).

BMI, body mass index; GFR, glomerular filtration rate; NDR, no diabetic retinopathy; PDR, proliferative diabetic retinopathy; SDR, simple diabetic retinopathy. 
Table 2 Circulating PUFAs of the study patients

\begin{tabular}{lccccc}
\hline Fatty acids, $\mu \mathrm{g} / \mathrm{mL}$ & All $(n=190)$ & NDR $(n=163)$ & SDR $(n=13)$ & PDR $(n=14)$ & $p$-value \\
\hline Linoleic acid (C18:2n6) & $868.1(370.9)$ & $875.9(363.5)$ & $792.5(538.3)$ & $847.6(281.1)$ & 0.723 \\
$\gamma$-Linolenic acid (C18:3n6) & $5.2(4.7)$ & $5.3(4.8)$ & $6.1(3.5)$ & $3.6(4.3)$ & 0.347 \\
Dihomo-gamma-linolenic acid (C20:3n6) & $27.6(10.7)$ & $28.3(11.0)$ & $24.4(9.7)^{*}$ & $21.8(6.2)^{*, \dagger}$ & 0.032 \\
\hline Logarithm of dihomo-gamma-linolenic acid & $8.24(2.23)$ & $8.40(2.27)$ & $7.58(2.10)^{*}$ & $7.05(1.36)^{*, \dagger}$ & 0.049 \\
Arachidonic acid (C20:4n6) & $157.6(69.6)$ & $160.4(68.6)$ & $165.6(68.6)$ & $117.1(75.2)$ & 0.074 \\
$\alpha$-Linolenic acid (C18:3n3) & $22.2(10.4)$ & $22.4(10.7)$ & $20.7(8.2)$ & $21.9(8.9)$ & 0.842 \\
Eicosatrienoic acid (C20:3n3) & $0.1(0.9)$ & $0.1(0.9)$ & $0.0(0.0)$ & $0.0(0.0)$ & 0.921 \\
\hline Eicosapentaenoic acid (C20:5n3) & $42.0(24.5)$ & $42.8(29.0)$ & $32.1(14.7)$ & $41.6(14.4)$ & 0.402 \\
Docosahexaenoic acid (C22:6n3) & $146.1(63.9)$ & $148.1(65.4)$ & $130.0(68.6)$ & $138.0(36.9)$ & 0.551 \\
\hline
\end{tabular}

Data are expressed as mean (SD).

$p$ values by one-way analysis of variance analyses. The analyses of circulating PUFA levels among three groups were performed by Tukey HSD test: ${ }^{*}, p<0.05$ versus $\mathrm{NDR} ;{ }^{\dagger}, p<0.05$ versus $\mathrm{SDR}$.

NDR, no diabetic retinopathy; PDR, proliferative diabetic retinopathy; PUFAs, polyunsaturated fatty acids; SDR, simple diabetic retinopathy.

C18:2n6

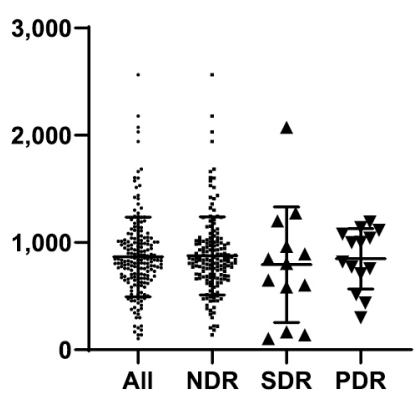

C18:3n3

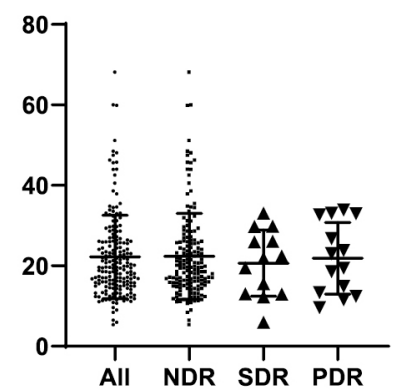

C18:3n6

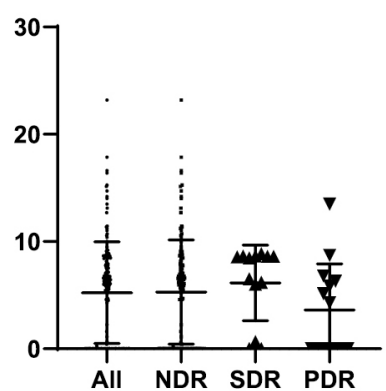

C20:3n3

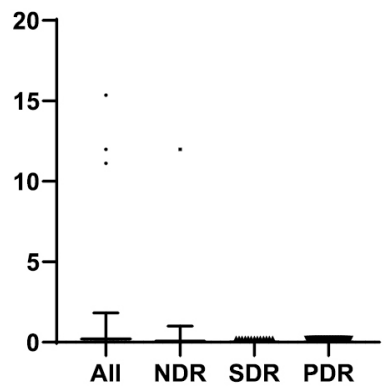

C20:3n6

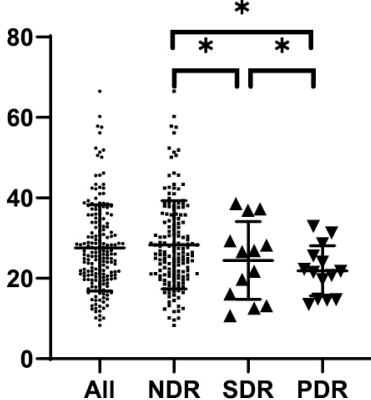

C20:5n3

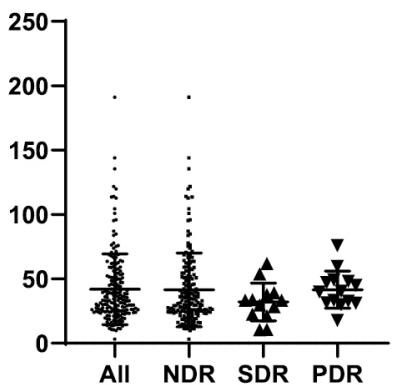

C20:4n 6

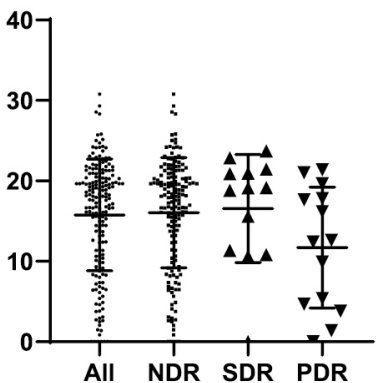

C22:6n3

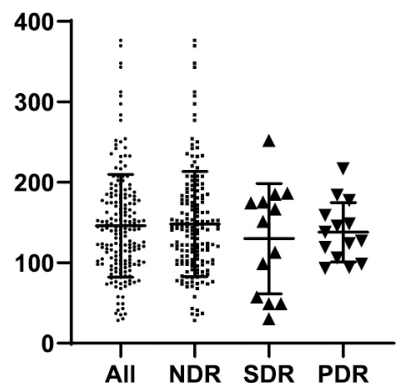

Fig. 2 Circulating PUFAs of the study patients

The analyses of circulating PUFA levels among three groups were performed by one-way ANOVA;,$p<0.05$.

NDR, no diabetic retinopathy; PDR, proliferative diabetic retinopathy; PUFAs, polyunsaturated fatty acids; SDR, simple diabetic retinopathy.

\section{Discussion}

In the present study of 190 Japanese individuals with T2DM from the KAMOGAWA-DM cohort study, we investigated the association between circulating $n-3$ or n-6 PUFAs and DR, and revealed that circulating DGLA is negatively associated with the presence of DR.

Possible explanations of the inverse association between circulating DGLA levels and the presence of DR were explored. DGLA, an essential fatty acid, is n-6 PUFA with 20 carbons derived in vivo from linolenic acid. DGLA is converted to another n-6 PUFA with 20 
Table 3 Logistic regression analyses for diabetic retinopathy

\begin{tabular}{|c|c|c|c|c|}
\hline & \multicolumn{2}{|l|}{ Univariate } & \multicolumn{2}{|c|}{ Multivariate } \\
\hline & Odds ratio $(95 \% \mathrm{CI})$ & $p$-value & Odds ratio $(95 \% \mathrm{CI})$ & $p$-value \\
\hline Men & $1.15(0.51-2.59)$ & 0.745 & $1.54(0.46-5.16)$ & 0.484 \\
\hline Disease duration, yrs & $1.05(1.01-1.10)$ & 0.015 & $1.04(0.99-1.10)$ & 0.117 \\
\hline Systolic blood pressure, $\mathrm{mmHg}$ & $1.02(0.99-1.04)$ & 0.100 & $1.02(0.99-1.04)$ & 0.143 \\
\hline Hemoglobin A1c, \% & $1.35(1.08-1.69)$ & 0.009 & $1.23(0.94-1.61)$ & 0.145 \\
\hline Triglycerides, mmol/L & $0.62(0.34-1.14)$ & 0.124 & $0.71(0.29-1.75)$ & 0.447 \\
\hline Total cholesterol, $\mathrm{mmol} / \mathrm{L}$ & $0.83(0.55-1.25)$ & 0.371 & $0.84(0.49-1.46)$ & 0.552 \\
\hline HDL-cholesterol, mmol/L & $1.23(0.53-2.88)$ & 0.631 & $0.91(0.25-3.30)$ & 0.888 \\
\hline Omega- 6 fatty acids, $\mu \mathrm{g} / \mathrm{mL}$ & $0.99(0.99-1.00)$ & 0.425 & $1.00(0.99-1.00)$ & 0.335 \\
\hline Never smoker & 1.00 (Reference) & - & 1.00 (Reference) & - \\
\hline Ex-smoker & $1.22(0.37-4.04)$ & 0.746 & $2.28(0.64-8.19)$ & 0.205 \\
\hline Current smoker & $2.84(1.13-7.13)$ & 0.026 & $0.92(0.20-4.12)$ & 0.912 \\
\hline Total energy, kcal/day & $1.00(0.99-1.00)$ & 0.571 & $0.84(0.49-1.46)$ & 0.889 \\
\hline Statin usage & $2.08(0.87-4.93)$ & 0.098 & $2.15(0.76-6.03)$ & 0.147 \\
\hline Log dihomo-gamma-linolenic acid & $0.76(0.62-0.96)$ & 0.020 & $0.72(0.53-0.99)$ & 0.038 \\
\hline
\end{tabular}

Multivariate analysis was adjusted for was adjusted for sex, disease duration, systolic blood pressure, hemoglobin A1c, triglycerides, total cholesterol, high-density lipoprotein cholesterol, omega-6 fatty acids, smoking status, total energy, and statin usage.

carbons, arachidonic acid (AA) [32, 33]. Both DGLA and AA are substrates of the lipid-peroxidizing enzyme cyclooxygenase (COX) which metabolizes DGLA and AA to various bioactive metabolites, such as the 1 and 2 series prostaglandins (PGs1 and PGs2), through a series of free radical reactions. PGs2 are generally known as pro-inflammatory metabolites, whereas PGs1 possess anti-inflammatory effects [34]. Prostaglandins E1 (PGE1), which is a type of PGs1, could inhibit the proliferation of vascular smooth muscle cells and the adhesion of vascular cells, and the development of atherosclerosis $[35,36]$. Furthermore, DGLA is metabolized by the 15 lipoxygenase into 15-(S)-hydroxy-8,11,13-eicosatrienoic acid (15-HETrE) [37], which has been reported to reduce the development of DR because it inhibits the activity of vasoconstrictors and metabolites of AA including thromboxanes (TXs) and leukotrienes (LTs) $[38,39]$. Reduced plasma and vitreal concentrations of PGE1, as well as increased levels of TXs and LTs have been reported in patients with DR [40-42]. Therefore, the imbalance between the vasodilators and platelet antiaggregators (e.g. PGE1) and the vasoconstrictors and platelet aggregators (e.g. TXs and LTs) may lead to ischemic vasoconstriction of retinal vessels and enhanced platelet aggregation.

Thus, the oxidative metabolites of DGLA, including PGE1 and 15-HETrE, have demonstrated clinical effi- cacy in a variety of diseases including suppressing chronic inflammation [37], promoting vasodilation [43], lowering blood pressure [44], and inhibiting smooth muscle cell proliferation associated with atherosclerotic plaque development [45]. In this study, circulating levels of DGLA in patients with DR were significantly lower than those without (patients with DR: $7.31 \pm 1.74$, patients without DR: $8.40 \pm 2.27, p=0.018$ ). Moreover, the logarithm of circulating DGLA levels and the logarithm of urine albumin excretion tended to be negatively correlated with each other $(\beta=-0.100, p=0.171)$. As mentioned above, the oxidative metabolites of DGLA, including PGE1 and 15-HETrE, have ameliorative effects on various diseases, and a similar mechanism was thought to have occurred in nephropathy.

Our study has a strength, and that is that the results of fatty acid measurements are extremely robust. At first, all results were normalized to the peak height of the internal standard $\mathrm{C} 17: 0$, and the results were quite robust. Average $\mathrm{CV}$ of three samples of intra-day was $0.0245 \%(0.004-0.039 \%)$ and average CV of six samples of inter-day precision was $0.00237 \%(0.0010-0.0033 \%)$ and linearity was from $0.56 \mu \mathrm{g} / \mathrm{mL}$ to $60.00 \mu \mathrm{g} / \mathrm{mL}$ (Supplementary Table 1). There are three major limitations in this study that could be addressed in future research. First, this study was a cross-sectional design, and further research should be conducted to establish the 
causal relationship between low circulating DGLA levels and DR. Second, the sample size in this study was relatively small and future large-scale studies are warranted. Third, fatty acids compete with each other in the metabolism, absorption, and incorporation to cells, therefore, it was better to use the proportion of specific fatty acid to total fatty acids to total fatty acids. However, many previous reports have performed various assessments using absolute values of circulating fatty acids [46-49], and significant associations with various diseases have been shown in those reports. Fourth, omega- 6 fatty acids are metabolized by a single metabolic pathway, and it is unclear why only DGLA was significantly associated with the presence of DR. This is a topic for future research.

In conclusion, this study was the first to demonstrate that circulating levels of DGLA in patients with DR were significantly lower than those without. It is important to conduct future prospective study to investigate the relationship between circulating DGLA levels and incident $\mathrm{DR}$, and explore the mechanisms that contribute to the difference between dietary and circulating DGLA.

\section{Acknowledgments}

We thank all of the staff members in Kyoto Prefectural University of Medicine. We would like to thank Editage (www.editage.com) for English language editing.

\section{Author Disclosures}

Yoshitaka Hashimoto has received grants from Asahi Kasei Pharma, personal fees from Daiichi Sankyo Co., Ltd., personal fees from Mitsubishi Tanabe Pharma Corp., personal fees from Sanofi K.K., personal fees from Novo Nordisk Pharma Ltd., outside the submitted work. Takafumi Senmaru has received personal fees from Ono Pharma Co., Ltd., Mitsubishi Tanabe Pharma Co, Astellas Pharma Inc., Kyowa Hakko Kirin Co., Ltd., Sanofi K.K., MSD K.K., Kowa Pharma Co., Ltd., Taisho Toyama Pharma Co., Ltd., Takeda Pharma Co., Ltd., Kissei Pharma Co., Ltd., Novo Nordisk Pharma Ltd., Eli Lilly Japan K.K. outside the submitted work. Emi Ushigome has received grants from the Japanese Study Group for Physiology and Management of Blood Pressure, the Astellas Foundation for Research on Metabolic Disorders (Grant number: 4024). Donated Fund Laboratory of Diabetes therapeutics is an endowment department, supported with an unrestricted grant from Ono Pharmaceutical Co., Ltd., and received personal fees from AstraZeneca plc, Astellas Pharma Inc., Daiichi Sankyo Co., Ltd., Kyowa Hakko Kirin
Company Ltd., Kowa Pharmaceutical Co., Ltd., MSD K.K., Mitsubishi Tanabe Pharma Corp., Novo Nordisk Pharma Ltd., Taisho Toyama Pharmaceutical Co., Ltd., Takeda Pharmaceutical Co., Ltd., Nippon Boehringer Ingelheim Co., Ltd., and Sumitomo Dainippon Pharma Co., Ltd., outside the submitted work. Masahide Hamaguchi has received grants from Asahi Kasei Pharma, Nippon Boehringer Ingelheim Co., Ltd., Mitsubishi Tanabe Pharma Corporation, Daiichi Sankyo Co., Ltd., Sanofi K.K., Takeda Pharmaceutical Company Limited, Astellas Pharma Inc., Kyowa Kirin Co., Ltd., Sumitomo Dainippon Pharma Co., Ltd., Novo Nordisk Pharma Ltd., and Eli Lilly Japan K.K., outside the submitted work. Mai Asano received personal fees from Novo Nordisk Pharma Ltd., Abbott Japan Co., Ltd., AstraZeneca plc, Kowa Pharmaceutical Co., Ltd., Ono Pharmaceutical Co., Ltd., Takeda Pharmaceutical Co., Ltd., outside the submitted work. Masahiro Yamazaki reports personal fees from MSD K.K., Sumitomo Dainippon Pharma Co., Ltd., Kowa Company, Limited, AstraZeneca PLC, Takeda Pharmaceutical Company Limited, Kyowa Hakko Kirin Co., Ltd., Daiichi Sankyo Co., Ltd., Kowa Pharmaceutical Co., Ltd., Ono Pharma Co., Ltd., outside the submitted work. Michiaki Fukui has received grants from Nippon Boehringer Ingelheim Co., Ltd., Kissei Pharma Co., Ltd., Mitsubishi Tanabe Pharma Co, Daiichi Sankyo Co., Ltd., Sanofi K.K., Takeda Pharma Co., Ltd., Astellas Pharma Inc., MSD K.K., Kyowa Hakko Kirin Co., Ltd., Sumitomo Dainippon Pharma Co., Ltd., Kowa Pharmaceutical Co., Ltd., Novo Nordisk Pharma Ltd., Ono Pharma Co., Ltd., Sanwa Kagaku Kenkyusho Co., Ltd. Eli Lilly Japan K.K., Taisho Pharma Co., Ltd., Terumo Co., Teijin Pharma Ltd., Nippon Chemiphar Co., Ltd., and Johnson \& Johnson K.K. Medical Co., Abbott Japan Co., Ltd., and received personal fees from Nippon Boehringer Ingelheim Co., Ltd., Kissei Pharma Co., Ltd., Mitsubishi Tanabe Pharma Corp., Daiichi Sankyo Co., Ltd., Sanofi K.K., Takeda Pharma Co., Ltd., Astellas Pharma Inc., MSD K.K., Kyowa Kirin Co., Ltd., Sumitomo Dainippon Pharma Co., Ltd., Kowa Pharma Co., Ltd., Novo Nordisk Pharma Ltd., Ono Pharma Co., Ltd., Sanwa Kagaku Kenkyusho Co., Ltd., Eli Lilly Japan K.K., Taisho Pharma Co., Ltd., Bayer Yakuhin, Ltd., AstraZeneca K.K., Mochida Pharma Co., Ltd., Abbott Japan Co., Ltd., Medtronic Japan Co., Ltd., Arkley Inc., Teijin Pharma Ltd. and Nipro Cor., outside the submitted work.

\section{Funding Statement}

None. 


\section{References}

1. Simó-Servat O, Hernández C, Simó R (2019) Diabetic retinopathy in the context of patients with diabetes. Ophthalmic Res 62: 211-217.

2. Maniadakis N, Konstantakopoulou E (2019) Cost effectiveness of treatments for diabetic retinopathy: a systematic literature review. Pharmacoeconomics 37: 995-1010.

3. Sacks FM, Hermans MP, Fioretto P, Valensi P, Davis T, et al. (2014) Association between plasma triglycerides and high-density lipoprotein cholesterol and microvascular kidney disease and retinopathy in type 2 diabetes mellitus: a global case-control study in 13 countries. Circulation 129: 999-1008.

4. Chew EY, Klein ML, Ferris FL, Remaley NA, Murphy RP, et al. (1996) Association of elevated serum lipid levels with retinal hard exudate in diabetic retinopathy: early treatment diabetic retinopathy study (ETDRS) report 22. Arch Ophthalmol 114: 1079-1084.

5. Kennedy A, Martinez K, Chuang C-C, LaPoint K, McIntosh M (2009) Saturated fatty acid-mediated inflammation and insulin resistance in adipose tissue: mechanisms of action and implications. J Nutr 139: 1-4.

6. Weisburger JH (1997) Dietary fat and risk of chromic disease: mechanistic insights from experimental studies. $J$ Am Diet Assoc 97: S16-S23.

7. Howard-Williams J, Patel P, Jelfs R, Carter RD, Awdry P, et al. (1985) Polyunsaturated fatty acids and diabetic retinopathy. Br J Ophthalmol 69: 15-18.

8. Houtsmuller AJ, Zahn KJ, Henkes HE (1980) Unsaturated fats and progression of diabetic retinopathy. Doc Ophthalmol 48: 363-371.

9. Sala-Vila A, Díaz-López A, Valls-Pedret C, Cofán M, García-Layana A, et al. (2016) Dietary marine $\omega-3$ fatty acids and incident sight-threatening retinopathy in middleaged and older individuals with type 2 diabetes: prospective investigation from the PREDIMED trial. JAMA Ophthalmol 134: 1142-1149.

10. Behl T, Kotwani A (2017) Omega-3 fatty acids in prevention of diabetic retinopathy. J Pharm Pharmacol 69: 946954.

11. Sapieha P, Chen J, Stahl A, Seaward MR, Favazza TL, et al. (2012) Omega-3 polyunsaturated fatty acids preserve retinal function in type 2 diabetic mice. Nutr Diabetes 2: e36.

12. Yee P, Weymouth AE, Fletcher EL, Vingrys AJ (2010) A role for omega-3 polyunsaturated fatty acid supplements in diabetic neuropathy. Investig Ophthalmol Vis Sci 51: 1755-1764.

13. Rubin D, Laposata M (1992) Cellular interactions between n-6 and n-3 fatty acids: a mass analysis of fatty acid elongation/desaturation, distribution among complex lipids, and conversion to eicosanoids. J Lipid Res 33: 1431-1440.

14. Harris WS, Mozaffarian D, Rimm E, Kris-Etherton P, Rudel LL, et al. (2009) Omega-6 fatty acids and risk for cardiovascular disease: a science advisory from the American Heart Association Nutrition Subcommittee of the Council on Nutrition, Physical Activity, and Metabolism; Council on Cardiovascular Nursing; and Council on Epidemiology and Prevention. Circulation 119: 902-907.

15. Wu JHY, Lemaitre RN, King IB, Song X, Psaty BM, et al. (2014) Circulating omega-6 polyunsaturated fatty acids and total and cause-specific mortality: the cardiovascular health study. Circulation 130: 1245-1253.

16. Li M, Xu C, Shi J, Ding J, Wan X, et al. (2018) Fatty acids promote fatty liver disease via the dysregulation of 3-mercaptopyruvate sulfurtransferase/hydrogen sulfide pathway. Gut 67: 2169-2180.

17. Tao M, McDowell MA, Saydah SH, Eberhardt MS (2008) Relationship of polyunsaturated fatty acid intake to peripheral neuropathy among adults with diabetes in the National Health and Nutrition Examination Survey (NHANES) 1999-2004. Diabetes Care 31: 93-95.

18. Sacks FM, Lichtenstein AH, Wu JHY, Appel LJ, Creager MA, et al. (2017) Dietary fats and cardiovascular disease: a presidential advisory from the American Heart Association. Circulation 136: e1-e23.

19. Sakai R, Hashimoto Y, Ushigome E, Miki A, Okamura T, et al. (2018) Late-night-dinner is associated with poor glycemic control in people with type 2 diabetes: the KAMOGAWA-DM cohort study. Endocr J 65: 395-402.

20. Expert Committee on the Diagnosis and Classification of Diabetes Mellitus. (2003) Report of the expert committee on the diagnosis and classification of diabetes mellitus. Diabetes Care 26 Suppl 1: S5-S20.

21. Kobayashi S, Murakami K, Sasaki S, Okubo H, Hirota N, et al. (2011) Comparison of relative validity of food group intakes estimated by comprehensive and brief-type selfadministered diet history questionnaires against $16 \mathrm{~d}$ dietary records in Japanese adults. Public Health Nutr 14: $1200-1211$.

22. Ministry of Education, Culture, Sports, Science and Technology. STANDARD TABLES OF FOOD COMPOSITION IN JAPAN - 2015 - (Seventh Revised Version). 2015 (In Japanse).

23. Okamura T, Hamaguchi M, Bamba R, Nakajima $H$, Yoshimura Y, et al. (2020) Immune modulating effects of additional supplementation of estradiol combined with testosterone in murine testosterone-deficient NAFLD model. Am J Physiol Liver Physiol 318: G989-G999.

24. Klein R, Klein BE, Moss SE, Davis MD, Demets DL (1984) The Wisconsin epidemiologic study of diabetic retinopathy: II. Prevalence and risk of diabetic retinopathy when age at diagnosis is less than 30 years. Arch Ophthalmol 102: 520-526.

25. Teuscher A, Schnell H, Wilson PW (1988) Incidence of diabetic retinopathy and relationship to baseline plasma glucose and blood pressure. Diabetes Care 11: 246-251.

26. Fu Z, Chen CT, Cagnone G, Heckel E, Sun Y, et al. 
(2019) Dyslipidemia in retinal metabolic disorders. EMBO Mol Med 11: e10473.

27. Gong Y, Fu Z, Liegl R, Chen J, Hellström A, et al. (2017) $\omega-3$ and $\omega-6$ long-chain PUFAs and their enzymatic metabolites in neovascular eye diseases. Am J Clin Nutr 106: $16-26$

28. Schnebelen C, Viau S, Grégoire S, Joffre C, CreuzotGarcher CP, et al. (2009) Nutrition for the eye: different susceptibility of the retina and the lacrimal gland to dietary omega- 6 and omega- 3 polyunsaturated fatty acid incorporation. Ophthalmic Res 41: 216-224.

29. Moss SE, Klein R, Klein BEK (1991) Association of cigarette smoking with diabetic retinopathy. Diabetes Care 14: $119-126$.

30. Wong MYZ, Man REK, Fenwick EK, Gupta P, Li LJ, et al. (2018) Dietary intake and diabetic retinopathy: a systematic review. PLoS One 13: e0186582.

31. Yau JWY, Rogers SL, Kawasaki R, Lamoureux EL, Kowalski JW, et al. (2012) Global prevalence and major risk factors of diabetic retinopathy. Diabetes Care 35: 556-564.

32. Ruan KH, Cervantes V, So SP (2009) Engineering of a novel hybrid enzyme: an anti-inflammatory drug target with triple catalytic activities directly converting arachidonic acid into the inflammatory prostaglandin E2. Protein Eng Des Sel 22: 733-740.

33. Kapoor R, Huang YS (2006) Gamma linolenic acid: an antiinflammatory omega-6 fatty acid. Curr Pharm Biotechnol 7: 531-534.

34. Levin G, Duffin KL, Obukowicz MG, Hummert SL, Fujiwara H, et al. (2002) Differential metabolism of dihomo-gamma-linolenic acid and arachidonic acid by cyclo-oxygenase-1 and cyclo-oxygenase-2: implications for cellular synthesis of prostaglandin E1 and prostaglandin E2. Biochem J 365: 489-496.

35. Gupta V, Davis M, Hope-Weeks LJ, Ahsan F (2011) PLGA microparticles encapsulating prostaglandin E1hydroxypropyl- $\beta$-cyclodextrin (PGE1-HP $\beta C D)$ complex for the treatment of pulmonary arterial hypertension (PAH). Pharm Res 28: 1733-1749.

36. Li JH, Yang P, Li AL, Wang Y, Ke YN, et al. (2011) Cardioprotective effect of liposomal prostaglandin E1 on a porcine model of myocardial infarction reperfusion noreflow. J Zhejiang Univ Sci B 12: 638-643.

37. Simopoulos AP (2008) The importance of the omega-6/ omega-3 fatty acid ratio in cardiovascular disease and other chronic diseases. Exp Biol Med 233: 674-688.

38. Iversen L, Fogh K, Bojesen G, Kragballe K (1991) Linoleic acid and dihomogammalinolenic acid inhibit leukotriene B4 formation and stimulate the formation of their 15-lipoxygenase products by human neutrophils in vitro. Evidence of formation of antiinflammatory compounds. Agents Actions 33: 286-291.

39. Chapkin RS, Miller CC, Somers SD, Erickson KL (1988) Ability of 15-hydroxyeicosatrienoic acid (15-OH-20: 3) to modulate macrophage arachidonic acid metabolism. Biochem Biophys Res Commun 153: 799-804.

40. Naveh N, Belkin M, Ben-Chaim O, Weissman C, Treister G (1990) Prostanoids in the vitreous of diabetic and nondiabetic human eyes with retinal detachment. Ophthalmic Res 22: 3-11.

41. Douros S, Phillips BA, Nadel A, Obstbaum SA (2001) Human vitreal prostaglandin levels and proliferative diabetic retinopathy. Doc Ophthalmol 103: 27-34.

42. Hardy P, Beauchamp M, Sennlaub F, Gobeil F, Tremblay $\mathrm{L}$, et al. (2005) New insights into the retinal circulation: inflammatory lipid mediators in ischemic retinopathy. Prostaglandins Leukot Essent Fat Acids 72: 301-325.

43. Ney P, Feelisch M (1995) Vasodilator effects of PGE1 in the coronary and systemic circulation of the rat are mediated by ATP-sensitive potassium $(\mathrm{K}+)$ channels. Agents Actions 45: 71-76.

44. Wendling MG, DuCharme DW (1974) Effects of chronic administration of prostaglandin E1 (PGE1) on arterial blood pressure of unanesthetized hypertensive rats. Prostaglandins 7: 71-82.

45. Mallat Z, Nakamura T, Ohan J, Lesèche G, Tedgui A, et al. (1999) The relationship of hydroxyeicosatetraenoic acids and F2-isoprostanes to plaque instability in human carotid atherosclerosis. J Clin Invest 103: 421-427.

46. Fekete K, Györei E, Lohner S, Verduci E, Agostoni C, et al. (2015) Long-chain polyunsaturated fatty acid status in obesity: a systematic review and meta-analysis. Obes Rev 16: 488-497.

47. Alhazmi A, Stojanovski E, Garg ML, McEvoy M (2014) Fasting whole blood fatty acid profile and risk of type 2 diabetes in adults: a nested case control study. PLoS One 9: e97001.

48. Ouchi S, Miyazaki T, Shimada K, Sugita Y, Shimizu M, et al. (2017) Decreased circulating dihomo-gammalinolenic acid levels are associated with total mortality in patients with acute cardiovascular disease and acute decompensated heart failure. Lipids Health Dis 16: 150.

49. Ouchi S, Miyazaki T, Shimada K, Sugita Y, Shimizu M, et al. (2017) Low docosahexaenoic acid, dihomo-gammalinolenic acid, and arachidonic acid levels associated with long-term mortality in patients with acute decompensated heart failure in different nutritional statuses. Nutrients 9: 956. 